Supplement of Solid Earth, 5, 693-704, 2014

http://www.solid-earth.net/5/693/2014/

doi:10.5194/se-5-693-2014-supplement

(C) Author(s) 2014. CC Attribution 3.0 License.

(c) (i)

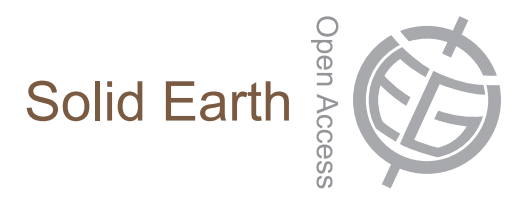

Supplement of

\title{
Physicochemical changes in pyrogenic organic matter (biochar) after 15 months of field aging
}

A. Mukherjee et al.

Correspondence to: A. R. Zimmerman (azimmer@ufl.edu) 
Supplemental Table S1. Characteristics of soils used in this study.

\begin{tabular}{lcclccc}
\hline Soil & latitude & longitude & Soil Series & \% sand & \% silt & \% clay \\
\hline BY soil $^{\text {a }}$ & 34.4836 & -84.3227 & Edneyville loam & 63 & 27 & 10 \\
PR soil $^{\text {b }}$ & 29.6833 & -82.0333 & Tavares or Candler series & 95 & 3 & 2 \\
\hline
\end{tabular}

\footnotetext{
${ }^{\mathrm{b}}$ Plant Science Research and Education Unit, Citra, FL. This site had not been fertilized or grazed for the past 10 yr. Soils were deep and extremely well-drained fine sands of the Tavares or Candler series (sandy hyperthermic, uncoated Typic Quartzipsamments)
} 
Supplemental Table S2. Elemental composition of fresh and aged biochars and soil/biochar mixtures.

\begin{tabular}{|c|c|c|c|c|c|c|c|c|c|c|c|c|c|c|c|}
\hline \multirow{2}{*}{$\begin{array}{l}\text { Biochar or } \\
\text { Soil/Biochar }\end{array}$} & $\mathrm{C}^{\mathrm{a}}$ & $\mathrm{N}^{\mathrm{a}}$ & $\mathrm{H}^{\mathrm{a}}$ & $\mathrm{O}^{\mathrm{b}}$ & $\mathrm{P}^{\mathrm{c}}$ & $\mathrm{K}^{\mathrm{c}}$ & $\mathrm{Mg}^{\mathrm{C}}$ & $\mathrm{Ca}^{\mathrm{c}}$ & $S^{c}$ & $\mathrm{~B}^{\mathrm{c}}$ & $\mathrm{Zn}^{\mathrm{c}}$ & $\mathrm{Mn}^{\mathrm{c}}$ & $\mathrm{Fe}^{\mathrm{c}}$ & $\mathrm{Cu}^{\mathrm{c}}$ & \multirow[t]{2}{*}{$\mathrm{O} / \mathrm{C}$} \\
\hline & \multicolumn{14}{|c|}{$\left(\mathrm{mg} \mathrm{g}^{-1}\right)$} & \\
\hline Fresh Oak-250 & $626 \pm 32$ & $1.9 \pm 0.3$ & $31 \pm 0.4$ & $342 \pm 32$ & 0.4 & 3.4 & 0.6 & 7.1 & 0.2 & 0.00 & 0.0 & 0.1 & 0.2 & 0.00 & 0.8 \\
\hline Fresh Oak-400 & $679 \pm 57$ & $3.7 \pm 0.7$ & $42 \pm 0.7$ & $276 \pm 57$ & 1.3 & 6.4 & 1.1 & 11.8 & 0.2 & 0.01 & 0.0 & 0.2 & 0.0 & 0.00 & 0.4 \\
\hline Fresh Oak-650 & $754 \pm 14$ & $4.6 \pm 0.4$ & $28 \pm 1.2$ & $213 \pm 15$ & 0.9 & 6.3 & 0.5 & 10.3 & 0.1 & 0.00 & 0.0 & 0.1 & 0.0 & 0.00 & 0.2 \\
\hline Fresh Pine-250 & $624 \pm 4$ & $0.0 \pm 0.0$ & $26 \pm 0.7$ & $350 \pm 5$ & 0.1 & 0.6 & 0.5 & 2.4 & 0.1 & 0.00 & 0.0 & 0.1 & 0.0 & 0.01 & 0.7 \\
\hline Fresh Pine-400 & $758 \pm 7$ & $0.7 \pm 0.9$ & $37 \pm 0.4$ & $204 \pm 7$ & 0.1 & 1.0 & 0.7 & 4.7 & 0.2 & 0.00 & 0.0 & 0.1 & 0.0 & 0.00 & 0.4 \\
\hline Fresh Pine-650 & $552 \pm 0$ & $0.0 \pm 0.0$ & $33 \pm 0.4$ & $416 \pm 8$ & 0.1 & 0.5 & 0.2 & 2.7 & 0.1 & 0.00 & 0.0 & 0.1 & 0.0 & 0.00 & 0.2 \\
\hline Fresh Grass-250 & $494 \pm 31$ & $12 \pm 2$ & $36 \pm 1.0$ & $458 \pm 31$ & 1.4 & 5.0 & 3.0 & 8.2 & 0.6 & 0.00 & 0.1 & 0.1 & 0.1 & 0.01 & 0.8 \\
\hline Fresh Grass-400 & $523 \pm 4$ & $14 \pm 0.2$ & $46 \pm 0.1$ & $417 \pm 4$ & 4.2 & 15.3 & 3.7 & 9.9 & 0.6 & 0.01 & 0.2 & 0.2 & 0.1 & 0.01 & 0.6 \\
\hline Fresh Grass-650 & $557 \pm 5$ & $5.7 \pm 0.4$ & $30 \pm 1.0$ & $408 \pm 6$ & 3.3 & 7.9 & 5.8 & 16.9 & 0.6 & 0.01 & 0.2 & 0.3 & 0.2 & 0.01 & 0.5 \\
\hline Aged Oak-250 & $594 \pm 22$ & $2.5 \pm 0.3$ & $47 \pm 6.2$ & $343 \pm 20$ & 0.1 & 1.8 & 0.7 & 8.2 & 0.2 & 0.00 & 0.0 & 0.1 & 0.0 & 0.01 & 0.6 \\
\hline Aged Oak-400 & $710 \pm 19$ & $3.0 \pm 0.7$ & $50 \pm 0.6$ & $222 \pm 3$ & $\mathrm{~nm}$ & $\mathrm{~nm}$ & $\mathrm{~nm}$ & $\mathrm{~nm}$ & $\mathrm{~nm}$ & $\mathrm{~nm}$ & $\mathrm{~nm}$ & $\mathrm{~nm}$ & $\mathrm{~nm}$ & $\mathrm{~nm}$ & 0.3 \\
\hline Aged Oak-650 & $813 \pm 2$ & $2.8 \pm 1.2$ & $76 \pm 7.8$ & $304 \pm 32$ & 0.8 & 0.9 & 0.5 & 11.6 & 0.1 & 0.00 & 0.0 & 0.1 & 0.0 & 0.01 & 0.4 \\
\hline Aged Pine-250 & $560 \pm 53$ & $1.2 \pm 0.6$ & $56 \pm 4.2$ & $429 \pm 5$ & $\mathrm{~nm}$ & $\mathrm{~nm}$ & $\mathrm{~nm}$ & $\mathrm{~nm}$ & $\mathrm{~nm}$ & $\mathrm{~nm}$ & $\mathrm{~nm}$ & $\mathrm{~nm}$ & $\mathrm{~nm}$ & $\mathrm{~nm}$ & 0.8 \\
\hline Aged Pine-400 & $634 \pm 72$ & $1.4 \pm 0.4$ & $46 \pm 38$ & $379 \pm 3$ & $\mathrm{~nm}$ & $\mathrm{~nm}$ & $\mathrm{~nm}$ & $\mathrm{~nm}$ & $\mathrm{~nm}$ & $\mathrm{~nm}$ & $\mathrm{~nm}$ & $\mathrm{~nm}$ & $\mathrm{~nm}$ & $\mathrm{~nm}$ & 0.6 \\
\hline Aged Pine-650 & $780 \pm 101$ & $0.8 \pm 0.8$ & $90 \pm 21$ & $539 \pm 18$ & $\mathrm{~nm}$ & $\mathrm{~nm}$ & $\mathrm{~nm}$ & $\mathrm{~nm}$ & $\mathrm{~nm}$ & $\mathrm{~nm}$ & $\mathrm{~nm}$ & $\mathrm{~nm}$ & $\mathrm{~nm}$ & $\mathrm{~nm}$ & 0.7 \\
\hline Aged Grass-250 & $575 \pm 20$ & $9.8 \pm 1.2$ & $43 \pm 0.8$ & $379 \pm 16$ & 0.2 & 1.4 & 2.9 & 12.5 & 0.3 & 0.00 & 0.2 & 0.2 & 0.2 & 0.02 & 0.7 \\
\hline Aged Grass-400 & $635 \pm 8$ & $15 \pm 2.1$ & $27 \pm 34$ & $319 \pm 46$ & $\mathrm{~nm}$ & $\mathrm{~nm}$ & $\mathrm{~nm}$ & $\mathrm{~nm}$ & $\mathrm{~nm}$ & $\mathrm{~nm}$ & $\mathrm{~nm}$ & $\mathrm{~nm}$ & $\mathrm{~nm}$ & $\mathrm{~nm}$ & 0.5 \\
\hline Aged Grass-650 & $704 \pm 24$ & $15 \pm 1.8$ & $35 \pm 1.6$ & $238 \pm 14$ & 1.9 & 1.3 & 3.0 & 15.9 & 0.5 & 0.00 & 0.3 & 0.3 & 0.5 & 0.02 & 0.3 \\
\hline
\end{tabular}


Supplemental Table S2. Continued.

\begin{tabular}{|c|c|c|c|c|c|c|c|c|c|c|c|c|c|c|c|}
\hline \multirow{2}{*}{$\begin{array}{c}\text { Biochar or } \\
\text { Soil/Biochar Mix }\end{array}$} & $\mathrm{C}^{\mathrm{a}}$ & $\mathrm{N}^{\mathrm{a}}$ & $\mathrm{H}^{\mathrm{a}}$ & $\mathrm{O}^{\mathrm{b}}$ & $\mathrm{P}^{\mathrm{c}}$ & $\mathrm{K}^{\mathrm{c}}$ & $\mathrm{Mg}^{\mathrm{c}}$ & $\mathrm{Ca}^{\mathrm{c}}$ & $S^{c}$ & $\mathrm{~B}^{\mathrm{c}}$ & $\mathrm{Zn}^{\mathrm{c}}$ & $\mathrm{Mn}^{\mathrm{c}}$ & $\mathrm{Fe}^{\mathrm{c}}$ & $\mathrm{Cu}^{\mathrm{c}}$ & \multirow[t]{2}{*}{$\mathrm{O} / \mathrm{C}$} \\
\hline & \multicolumn{14}{|c|}{$\left(\mathrm{mg} \mathrm{g}^{-1}\right)$} & \\
\hline Aged BY & $27 \pm 4$ & $2.2 \pm 0.3$ & $4.7 \pm 0.7$ & $966 \pm 5$ & 1.1 & 0.2 & 0.4 & 5.7 & 0.2 & 0.0 & 0.0 & 0.2 & 1.7 & 0.0 & - \\
\hline BY/Aged Oak-250 & $49 \pm 6$ & $3.1 \pm 0.3$ & $7.1 \pm 0.1$ & $947 \pm 0$ & $\mathrm{~nm}$ & $\mathrm{~nm}$ & $\mathrm{~nm}$ & $\mathrm{~nm}$ & $\mathrm{~nm}$ & $\mathrm{~nm}$ & $\mathrm{~nm}$ & $\mathrm{~nm}$ & $\mathrm{~nm}$ & $\mathrm{~nm}$ & - \\
\hline BY/Aged Oak-650 & $54 \pm 4$ & $3.4 \pm 0.1$ & $7.9 \pm 0.3$ & $935 \pm 4$ & $\mathrm{~nm}$ & $\mathrm{~nm}$ & $\mathrm{~nm}$ & $\mathrm{~nm}$ & $\mathrm{~nm}$ & $\mathrm{~nm}$ & $\mathrm{~nm}$ & $\mathrm{~nm}$ & $\mathrm{~nm}$ & $\mathrm{~nm}$ & - \\
\hline Aged PR & $3.3 \pm 3.8$ & $0.2 \pm 0.2$ & $0.7 \pm 0.1$ & $991 \pm 1$ & 0.4 & 0.2 & 0.2 & 2.2 & 0.1 & 0.0 & 0.0 & 0.0 & 0.6 & 0.0 & - \\
\hline PR/Aged Oak-250 & $10 \pm 2$ & $0.4 \pm 0.1$ & $0.8 \pm 0.1$ & $987 \pm 0$ & $\mathrm{~nm}$ & $\mathrm{~nm}$ & $\mathrm{~nm}$ & $\mathrm{~nm}$ & $\mathrm{~nm}$ & $\mathrm{~nm}$ & $\mathrm{~nm}$ & $\mathrm{~nm}$ & $\mathrm{~nm}$ & $\mathrm{~nm}$ & - \\
\hline PR/Aged Oak-650 & $11 \pm 0.0$ & $0.5 \pm 0.0$ & $1.1 \pm 0.1$ & $993 \pm 1$ & $\mathrm{~nm}$ & $\mathrm{~nm}$ & $\mathrm{~nm}$ & $\mathrm{~nm}$ & $\mathrm{~nm}$ & $\mathrm{~nm}$ & $\mathrm{~nm}$ & $\mathrm{~nm}$ & $\mathrm{~nm}$ & $\mathrm{~nm}$ & - \\
\hline
\end{tabular}

Notes/Abbreviations:

nm: not measured

BY: agricultural Entisol, Gainesville, Florida, USA

PR: forest Spodosol, Marion County, Florida, USA

a: measured via high temperature catalyzed combustion (CHN analyzer) and calculated on a dry ash-free basis

b:calculated from the following equation: \% $\mathrm{O}=100-\%(\mathrm{C}+\mathrm{N}+\mathrm{H})$

C: analyzed on ICP after total digested acid extraction procedure (AOAC 985.01)

Note: The $\mathrm{O}$ concentration was calculated by weight difference, assuming biochars were consisted of $\mathrm{C}, \mathrm{H}, \mathrm{N}$, and $\mathrm{O}$ 
Supplemental Table S3. Physicochemical characteristics of fresh and aged biochars (average, $n=3$ ).

\begin{tabular}{|c|c|c|c|c|c|c|c|c|c|c|}
\hline \multirow{3}{*}{$\begin{array}{c}\text { Biochar or } \\
\text { Soil/Biochar Mix } \\
\text { Fresh Oak-250 }\end{array}$} & \multirow{3}{*}{$\begin{array}{l}\mathrm{pH} \\
3.5 \pm 0.0\end{array}$} & \multirow{2}{*}{\multicolumn{2}{|c|}{$\begin{array}{l}\text { VM } \\
\\
(\%)\end{array}$}} & \multicolumn{2}{|c|}{$\mathrm{SA}\left(\mathrm{m}^{2} \mathrm{~g}^{-1}\right)$} & \multirow{2}{*}{\multicolumn{2}{|c|}{$\begin{array}{lr}\text { CA } & \text { PA } \\
& \left(\mathrm{mmol} \mathrm{g}^{-1}\right)\end{array}$}} & \multirow{3}{*}{$\begin{array}{c}\text { TA } \\
8.1 \pm 2.4\end{array}$} & \multirow{2}{*}{\multicolumn{2}{|c|}{$\begin{array}{l}\text { CEC } \\
\qquad\left(\mathrm{cmol}_{\mathrm{c}} \mathrm{kg}^{-1}\right)\end{array}$}} \\
\hline & & & & \multirow{2}{*}{$\begin{array}{l}\mathrm{CO}_{2} \\
275 \pm 89\end{array}$} & \multirow{2}{*}{$\begin{array}{l}\mathrm{N}_{2} \\
1.2 \pm 0.8\end{array}$} & & & & & \\
\hline & & $66.0 \pm 4.4$ & $1.4 \pm 0.1$ & & & \multirow{2}{*}{$\begin{array}{l}5.0 \pm 0.1 \\
3.9 \pm 0.3\end{array}$} & \multirow{2}{*}{$\begin{array}{l}3.2 \pm 0.7 \\
2.4 \pm 0.3\end{array}$} & & \multirow{2}{*}{$\begin{array}{l}35.6 \pm 6.1 \\
10.7 \pm 2.3\end{array}$} & 0.0 \\
\hline Fresh Oak-400 & $6.7 \pm 0.2$ & $51.9 \pm 5.2$ & $2.6 \pm 0.2$ & $250 \pm 74$ & $2.0 \pm 1.2$ & & & $6.0 \pm 0.2$ & & 0.0 \\
\hline Fresh Oak-650 & $9.1 \pm 0.0$ & $36.4 \pm 1.1$ & $3.7 \pm 0.2$ & $533 \pm 42$ & $226 \pm 7.0$ & $3.9 \pm 0.1$ & $1.4 \pm 0.1$ & $4.7 \pm 0.1$ & $8.9 \pm 1.8$ & 0.0 \\
\hline Fresh Pine-250 & $3.1 \pm 0.1$ & $61.1 \pm 1.6$ & $0.3 \pm 0.1$ & $335 \pm 112$ & $0.3 \pm 0$ & $5.7 \pm 0.1$ & $0.4 \pm 0.4$ & $7.2 \pm 0.1$ & $45.2 \pm 2.1$ & 0.0 \\
\hline Fresh Pine-400 & $5.1 \pm 0.1$ & $58.6 \pm 1.0$ & $0.5 \pm 0.2$ & $329 \pm 122$ & $3.1 \pm 1.5$ & $4.9 \pm 0.1$ & $1.2 \pm 0.2$ & $6.1 \pm 0.1$ & $12.4 \pm 1.1$ & 0.0 \\
\hline Fresh Pine-650 & $6.8 \pm 0.1$ & $25.2 \pm 4.7$ & $1.1 \pm 0.1$ & $604 \pm 65$ & $254 \pm 104$ & $4.4 \pm 0.3$ & $0.1 \pm 0.0$ & $4.4 \pm 0.0$ & $10.4 \pm 2.2$ & 0.0 \\
\hline Fresh Grass-250 & $4.5 \pm 0.0$ & $62.5 \pm 2.9$ & $6.8 \pm 0.2$ & $222 \pm 75$ & $3.4 \pm 1.6$ & $6.2 \pm 0.0$ & $1.9 \pm 0.2$ & $8.0 \pm 0.1$ & $58.2 \pm 15.5$ & 0.0 \\
\hline Fresh Grass-400 & $7.8 \pm 0.0$ & $51.4 \pm 6.4$ & $13.2 \pm 0.2$ & $178 \pm 42$ & $6.0 \pm 6.2$ & $3.9 \pm 0.3$ & $1.9 \pm 0.0$ & $5.9 \pm 0.4$ & $18.2 \pm 6.9$ & 0.0 \\
\hline Fresh Grass-650 & $10.0 \pm 0.0$ & $33.0 \pm 1.2$ & $15.9 \pm 0.5$ & $433 \pm 82$ & $73 \pm 39$ & $3.9 \pm 0.1$ & $1.1 \pm 0.1$ & $4.7 \pm 0.0$ & $35.9 \pm 6.9$ & 0.0 \\
\hline Aged Oak-250 & $4.00 \pm 0.0$ & $66.1 \pm 0.9$ & $2.0 \pm 0.3$ & $208 \pm 17$ & $0.6 \pm 0.0$ & $1.9 \pm 0.3$ & $3.8 \pm 0.3$ & $4.7 \pm 0.3$ & $135.6 \pm 3.7$ & $94.3 \pm 72.5$ \\
\hline Aged Oak-400 & $5.60 \pm 0.0$ & $50.2 \pm 1.5$ & $2.2 \pm 0.2$ & $283 \pm 20$ & $0.7 \pm 0.0$ & $\mathrm{~nm}$ & $\mathrm{~nm}$ & $\mathrm{~nm}$ & $\mathrm{~nm}$ & $\mathrm{~nm}$ \\
\hline Aged Oak-650 & $6.7 \pm 0.3$ & $29.4 \pm 4.3$ & $2.9 \pm 0.8$ & $556 \pm 10$ & $35 \pm 19$ & $1.1 \pm 0.5$ & $0.8 \pm 0.0$ & $0.8 \pm 0.3$ & $110.6 \pm 25.3$ & $51.2 \pm 13.3$ \\
\hline Aged Pine-250 & $3.70 \pm 0.0$ & $63.7 \pm 2.3$ & $1.1 \pm 0.5$ & $249 \pm 2$ & $0.5 \pm 0.1$ & $\mathrm{~nm}$ & $\mathrm{~nm}$ & $\mathrm{~nm}$ & $\mathrm{~nm}$ & $\mathrm{~nm}$ \\
\hline Aged Pine-400 & $5.00 \pm 0.0$ & $63.2 \pm 2.5$ & $1.1 \pm 0.5$ & $222 \pm 14$ & $1.7 \pm 0.9$ & $\mathrm{~nm}$ & $\mathrm{~nm}$ & $\mathrm{~nm}$ & $\mathrm{~nm}$ & $\mathrm{~nm}$ \\
\hline Aged Pine-650 & $5.40 \pm 0.0$ & $24.7 \pm 1.4$ & $0.3 \pm 0.2$ & $577 \pm 10$ & $0.5 \pm 0.1$ & $\mathrm{~nm}$ & $\mathrm{~nm}$ & $\mathrm{~nm}$ & $\mathrm{~nm}$ & $\mathrm{~nm}$ \\
\hline Aged Grass-250 & $4.20 \pm 0.0$ & $65.2 \pm 1.4$ & $7.7 \pm 0.1$ & $238 \pm 17$ & $4.6 \pm 2.2$ & $2.2 \pm 0.0$ & $2.5 \pm 0.7$ & $4.2 \pm 0.3$ & $205.8 \pm 84.3$ & $75.3 \pm 33.8$ \\
\hline Aged Grass-400 & $6.00 \pm 0.0$ & $53.1 \pm 2.4$ & $8.2 \pm 0.5$ & $237 \pm 2$ & $1.8 \pm 0.1$ & $\mathrm{~nm}$ & $\mathrm{~nm}$ & $\mathrm{~nm}$ & $\mathrm{~nm}$ & $\mathrm{~nm}$ \\
\hline Aged Grass-650 & $7.00 \pm 0.0$ & $41.5 \pm 3.1$ & $10.0 \pm 0.3$ & $517 \pm 9$ & $39 \pm 8$ & $0.8 \pm 0.5$ & $1.2 \pm 0.5$ & $1.2 \pm 0.3$ & $238.2 \pm 22.7$ & $106.1 \pm 14.8$ \\
\hline
\end{tabular}

Notes/Abbreviations: $\mathrm{VM}=$ volatile matter, $\mathrm{AC}=$ ash content, $\mathrm{SA}=$ surface area as measured by $\mathrm{CO}_{2}$ and $\mathrm{N}_{2}$ sorptometry, respectively. $\mathrm{CA}=$ carboxylic acidity, PA = phenolic acidity, and TA = total acidity are surface acid functional groups as measured by Boehm titration (Boehm, 1964; Goertzen et al., 2010), CEC and AEC = cation and anion exchange capacity, respectively, measured at pH 6-7, nm = not measured 
Supplemental Table S4. ANOVA test ( $p$ values) for differences between means of biochar parameters for each treatment type; parent biomass (oak, pine and grass), condition (fresh and aged), and highest treatment temperature (HTT: 250, 400 and $650{ }^{\circ} \mathrm{C}$ ) and their interactive effects.

\begin{tabular}{|c|c|c|c|c|c|c|c|c|c|c|}
\hline \multirow{2}{*}{ Sources } & \multirow{2}{*}{$\mathrm{DF}$} & \multicolumn{9}{|c|}{ Analyses } \\
\hline & & $\mathrm{C} \%$ & $\mathrm{O} / \mathrm{C}$ & VM & Ash & $\mathrm{N}_{2}-\mathrm{SA}$ & $\mathrm{CO}_{2}-\mathrm{SA}$ & $\mathrm{pH}$ & $\mathrm{CEC}^{1}$ & $\mathrm{AEC}^{2}$ \\
\hline Biomass Type & 2 & 0.095 & 0.213 & 0.682 & 0.001 & 0.676 & 0.024 & 0.001 & 0.018 & 0.227 \\
\hline Condition & 1 & 0.198 & 0.548 & 0.216 & 0.130 & 0.050 & 0.297 & 0.001 & na & na \\
\hline Biomass. x Cond. & 2 & 0.429 & 0.070 & 0.089 & 0.129 & 0.452 & 0.072 & 0.009 & na & na \\
\hline HTT & 2 & 0.092 & 0.011 & $<0.001$ & 0.069 & 0.020 & $<0.001$ & $<0.001$ & 0.004 & 0.500 \\
\hline Biomass x HTT & 4 & 0.713 & 0.844 & 0.014 & 0.172 & 0.707 & 0.165 & 0.028 & na & na \\
\hline Cond. x HTT & 2 & 0.214 & 0.269 & 0.803 & 0.181 & 0.045 & 0.369 & 0.001 & na & na \\
\hline
\end{tabular}

Abbreviations: DF = Degree of Freedom, SA = Surface Area, VM = Volatile Matter, CEC = Cation Exchange Capacity at pH 7, AEC = Anion Exchange Capacity at $\mathrm{pH}$ 7, HTT = Highest Treatment Temperature

na: Not Applicable - Not enough data points to run ANOVA because those properties were only analyzed on four instead of nine aged biochar samples
1. $\mathrm{n}=18$
2. $\mathrm{n}=4$ 
Supplemental Table S5. Correlation coefficients $\left(\mathrm{R}^{2}\right)$ for linear relationships between various properties of aged biochars only. Underlined numbers are inverse correlations and bold numbers are significant at $p<0.05$.

\begin{tabular}{|c|c|c|c|c|c|c|c|c|}
\hline & \multicolumn{8}{|c|}{$\mathrm{R}^{2}$} \\
\hline & $\mathrm{N}_{2} \mathrm{SA}^{\mathrm{a}}$ & $\mathrm{CO}_{2} \mathrm{SA}^{\mathrm{a}}$ & $\mathrm{VM}^{\mathrm{a}}$ & $\operatorname{Ash}^{a}$ & $\mathrm{pH}^{\mathrm{a}}$ & $\mathrm{CEC}^{\mathrm{b}}$ & $\mathrm{AEC}^{\mathrm{b}}$ & $\mathrm{O} / \mathrm{C}^{\mathrm{a}}$ \\
\hline $\mathrm{CO}_{2} \mathrm{SA}^{\mathrm{a}}$ & $\underline{0.44}$ & & & & & & & \\
\hline $\mathrm{VM}^{\mathrm{a}}$ & $\overline{-0.26}$ & $\underline{-0.90}$ & & & & & & \\
\hline$A C^{a}$ & 0.21 & $\overline{0.00}$ & 0.00 & & & & & \\
\hline $\mathrm{pH}^{\mathrm{a}}$ & $\underline{0.56}$ & $\underline{0.46}$ & -0.52 & 0.19 & & & & \\
\hline $\mathrm{CEC}^{\mathrm{b}}$ & $\overline{0.07}$ & $\overline{0.01}$ & 0.01 & 0.72 & 0.08 & & & \\
\hline$A E C^{b}$ & 0.18 & 0.07 & -0.01 & 0.55 & 0.19 & $\underline{0.93}$ & & \\
\hline $\mathrm{O} / \mathrm{C}^{\mathrm{a}}$ & -0.37 & -0.10 & 0.14 & -0.15 & $\underline{-0.66}$ & $\overline{-0.14}$ & -0.33 & \\
\hline $\mathrm{C} \%^{\mathrm{a}}$ & 0.28 & $\underline{0.76}$ & $\underline{-0.90}$ & -0.02 & $\underline{0.59}$ & -0.05 & -0.00 & -0.28 \\
\hline
\end{tabular}

Notes/Abbreviations:

$\mathrm{SA}=$ surface area, $\mathrm{VM}=$ volatile matter, $\mathrm{AC}=$ ash content, $\mathrm{CEC}$ and AEC = cation and anion exchange capacity measured at pH 6-7. $\mathrm{a}: \mathrm{n}=9$

b: $\mathrm{n}=4$ 
Supplemental Table S6. Correlation coefficients $\left(R^{2}\right)$ between various properties of fresh biochars only $(n=9)$, negative signs indicate negative correlations. The underlined values are significant at $p<0.05$.

\begin{tabular}{|c|c|c|c|c|c|c|c|}
\hline & \multicolumn{7}{|c|}{$\mathrm{R}^{2}$} \\
\hline & $\mathrm{N}_{2}-\mathrm{SA}^{\mathrm{a}}$ & $\mathrm{CO}_{2}-\mathrm{SA}^{\mathrm{a}}$ & $\mathrm{VM}^{\mathrm{a}}$ & $\operatorname{Ash}^{a}$ & $\mathrm{pH}^{\mathrm{a}}$ & $\mathrm{CEC}^{\mathrm{a}}$ & $\mathrm{O} / \mathrm{C}^{\mathrm{a}}$ \\
\hline $\mathrm{CO}_{2}-\mathrm{SA}^{\mathrm{a}}$ & $\underline{0.84}$ & & & & & & \\
\hline $\mathrm{VM}^{\mathrm{a}}$ & $\underline{-0.73}$ & $\underline{-0.67}$ & & & & & \\
\hline$A C^{a}$ & -0.01 & -0.04 & -0.08 & & & & \\
\hline $\mathrm{pH}^{\mathrm{a}}$ & 0.27 & 0.17 & $\underline{-0.63}$ & 0.43 & & & \\
\hline $\mathrm{CEC}^{\mathrm{a}}$ & -0.23 & -0.18 & 0.24 & 0.06 & -0.19 & & \\
\hline $\mathrm{O} / \mathrm{C}^{\mathrm{a}}$ & $\underline{-0.61}$ & $\underline{-0.56}$ & $\underline{0.63}$ & 0.02 & -0.38 & $\underline{0.71}$ & \\
\hline $\mathrm{C} \%^{\mathrm{a}}$ & 0.01 & 0.05 & 0.00 & -0.29 & 0.00 & -0.37 & -0.22 \\
\hline
\end{tabular}

Notes/Abbreviations:

$\mathrm{SA}=$ surface area, $\mathrm{VM}=$ volatile matter, $\mathrm{AC}=$ ash content, $\mathrm{CEC}=$ cation exchange capacity at $\mathrm{pH} 7$ $\mathrm{a}: \mathrm{n}=9$ 
Supplemental Table S7. Correlation coefficients $\left(\mathrm{R}^{2}\right)$ between various properties of biochars (pooled fresh and aged together, $\mathrm{n}=18$ ). Negative values indicate inverse correlations. The underlined values are significant at $p<0.05$.

\begin{tabular}{|c|c|c|c|c|c|c|c|c|}
\hline & \multicolumn{8}{|c|}{$\mathrm{R}^{2}$} \\
\hline & $\mathrm{N}_{2}-\mathrm{SA}^{\mathrm{a}}$ & $\mathrm{CO}_{2}-\mathrm{SA}^{\mathrm{a}}$ & $\mathrm{VM}^{\mathrm{a}}$ & $\operatorname{Ash}^{a}$ & $\mathrm{pH}^{\mathrm{a}}$ & $\mathrm{CEC}^{\mathrm{a}}$ & $\mathrm{AEC}^{\mathrm{b}}$ & $\mathrm{O} / \mathrm{C}^{\mathrm{a}}$ \\
\hline $\mathrm{CO}_{2}-\mathrm{SA}^{\mathrm{a}}$ & $\underline{0.92}$ & & & & & & & \\
\hline $\mathrm{VM}^{\mathrm{a}}$ & $\underline{-0.83}$ & $\underline{-0.84}$ & & & & & & \\
\hline $\mathrm{AC}^{\mathrm{a}}$ & -0.01 & -0.00 & -0.02 & & & & & \\
\hline $\mathrm{pH}^{\mathrm{a}}$ & 0.34 & 0.33 & $\underline{-0.59}$ & 0.34 & & & & \\
\hline $\mathrm{CEC}^{\mathrm{a}}$ & -0.00 & 0.00 & 0.01 & 0.32 & 0.02 & & & \\
\hline$A E C^{b}$ & 0.02 & 0.03 & -0.01 & 0.32 & 0.10 & $\underline{0.93}$ & & \\
\hline $\mathrm{O} / \mathrm{C}^{\mathrm{a}}$ & -0.36 & -0.37 & $\underline{0.57}$ & -0.01 & $\underline{-0.71}$ & 0.04 & -0.00 & \\
\hline $\mathrm{C} \%{ }^{\mathrm{a}}$ & 0.36 & 0.34 & -0.30 & -0.15 & 0.23 & -0.12 & -0.01 & $\underline{-0.68}$ \\
\hline
\end{tabular}

Notes/Abbreviations:

$\mathrm{SA}=$ surface area, $\mathrm{VM}=$ volatile matter, $\mathrm{AC}=$ ash content, $\mathrm{CEC}=$ cation exchange capacity at $\mathrm{pH}$ 7, $\mathrm{AEC}=$ anion exchange capacity at $\mathrm{pH} 7$ $\mathrm{a}: \mathrm{n}=18$

b: $\mathrm{n}=4$ 


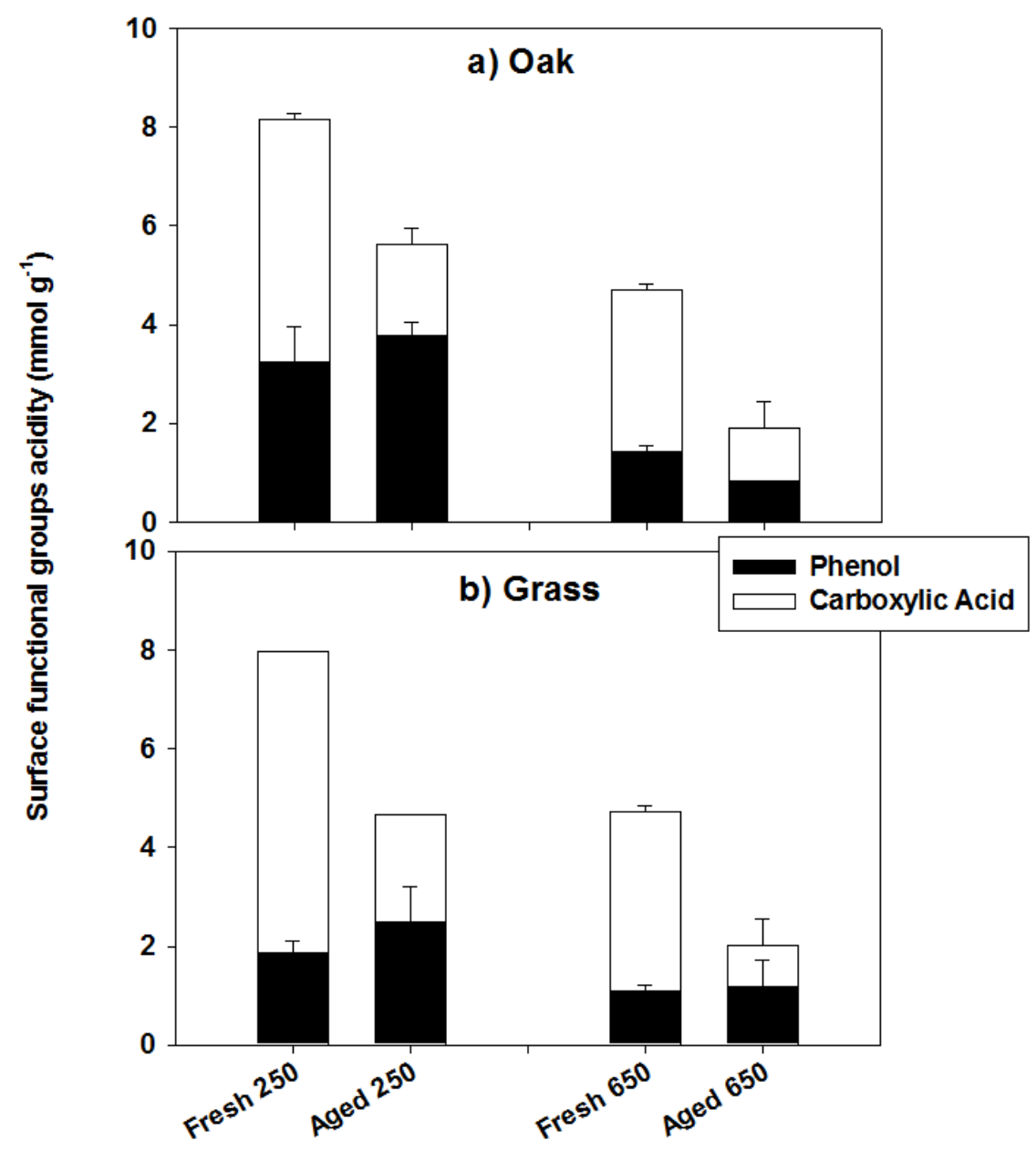

Supplemental Figure S1. Comparison between the acidic surface functional group content of fresh and aged a) oak and b) grass biochars produced at 250 and $650{ }^{\circ} \mathrm{C}$ determined using the Boehm titration method (Boehm, 1964; Goertzen et al., 2010) as detailed in Mukherjee et al. (2011). Error bar shows the standard deviation of three replicate analyses. This method was recently called into question for use with biochar by a study that showed that the presence of inorganic basic and acidic components in biochar (e.g. carbonates, oxides, silica) that can interfere with the titration results. (Tsechansky and Graber, 2014). Thus, the acidic surface functional group quantities may be invalid and are not presented in the main manuscript. 

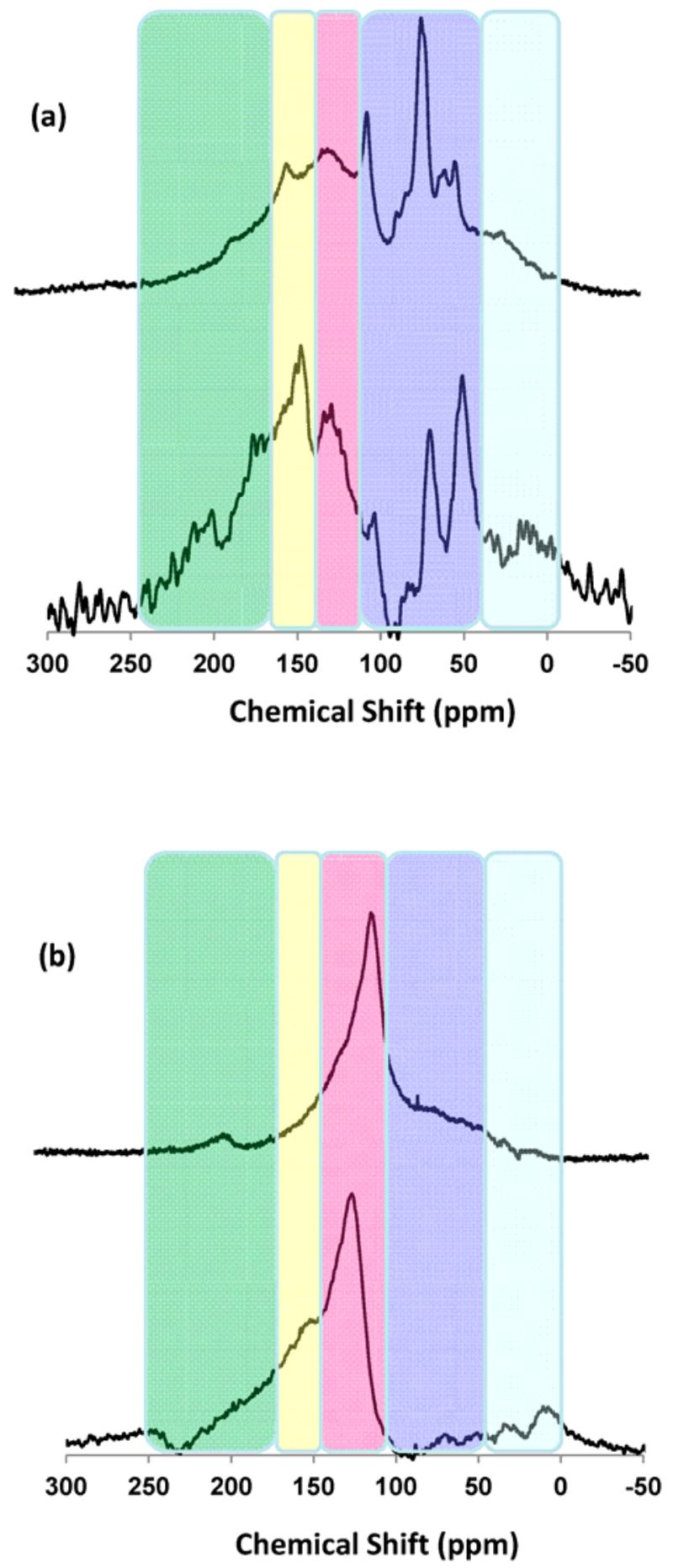

Supplemental Figure S2. ${ }^{13} \mathrm{C}$ CP-MAS NMR spectra of fresh (top) and aged (bottom) oak biochars prepared at (a) $250^{\circ}$ and (b) $650{ }^{\circ} \mathrm{C}$. Shaded regions correspond to spectral regions representing functional groups as described in text. 

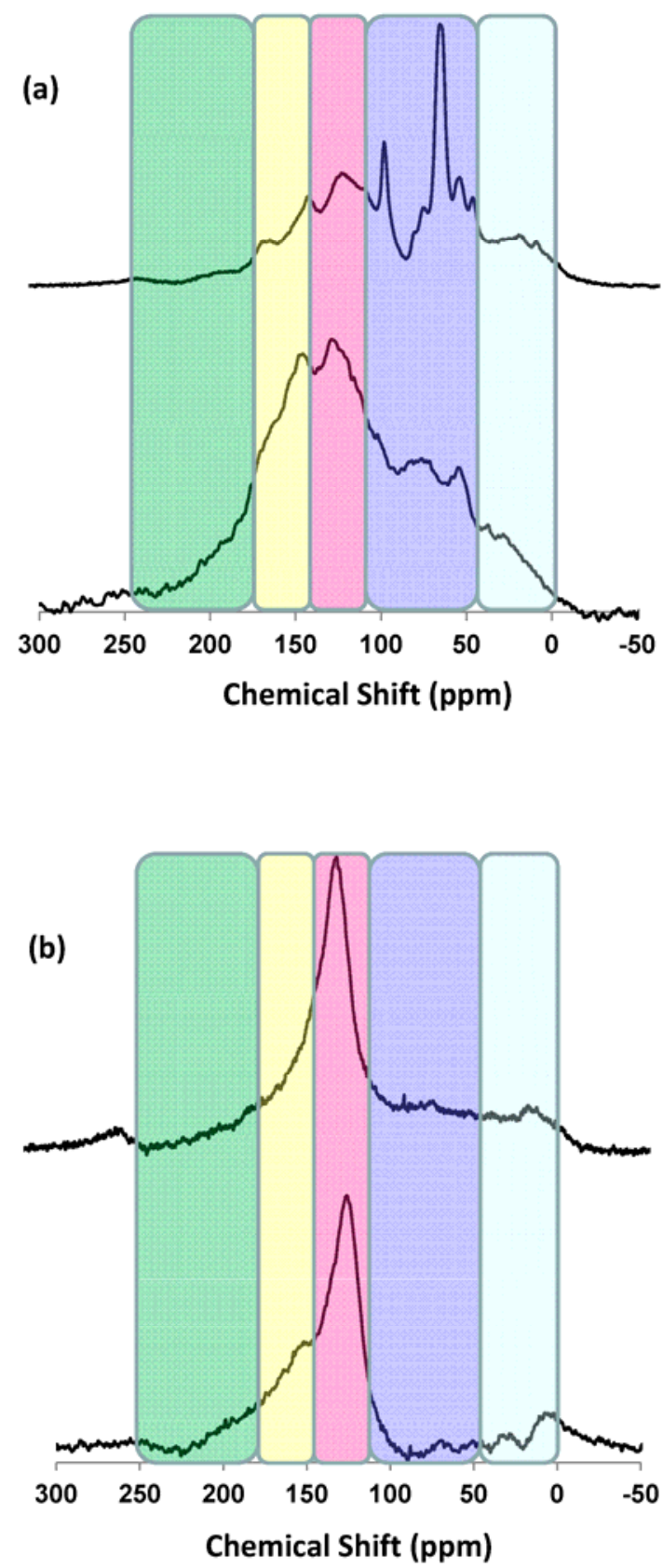

Supplemental Figure S3. ${ }^{13} \mathrm{C}$ CP-MAS NMR spectra of fresh (top) and aged (bottom) grass biochars prepared at (a) $250^{\circ}$ and (b) $650{ }^{\circ} \mathrm{C}$. Shaded regions correspond to spectral regions representing functional groups as described in text. 


\section{References for Supplemental Information Section}

Boehm, H.P., Diehl, E., Heck, W., and Sappok, R., 1964. Surface oxides of carbon. Angewandte Chemie International Edition 3, 669-677.

Goertzen, S.L., Thériault, K.D., Oickle, A.M., Tarasuk, A.C., Andreas, H.A., 2010.

Standardization of the Boehm titration. Part I. $\mathrm{CO}_{2}$ expulsion and endpoint determination. Carbon 48, 1252-1261.

Mukherjee, A., Zimmerman, A.R., Harris, W.G., 2011. Surface chemistry variations among a series of laboratory-produced biochars. Geoderma, 163, 247-255.

Tsechansky, L., Graber, E.R., 2014. Methodological limitations to determining acidic groups at biochar surfaces via the Boehm titration. Carbon 66, 730-733. 